Bibliotecas. Vol 37, № 1, enero - junio, 2019. EISSN: 1659-3286

URL: http://www.revistas.una.ac.cr/index.php/bibliotecas/index

DOI: http://dx.doi.org/10.15359/rb.37-1.2.

Licencia: Creative Commons (BY-NC-SA) 4.0 Internacional

\title{
Por qué estudiar bibliotecología y archivística: revisión de los motivos de elección profesional y de estrategias institucionales*
}

\author{
Why Study Librarianship and Archival Science: Review of Professional Choices and \\ Institutional Strategies
}

\author{
Ruth Helena Vallejo Sierra \\ Investigadora independiente
}

Recibido: 07 de setiembre de $2018 \quad$ Aceptado: 08 de marzo de 2019

Publicado: 19 de junio de 2019

\section{Resumen}

Este documento revisa las razones por las cuales se estudia Bibliotecología y Archivística y las estrategias institucionales para captar estudiantes. Se parte de un estudio general sobre la profesión, que considera no solo a estudiantes, sino a profesionales, expertos y empleadores a través de un estudio mixto que permite conocer la opinión de este grupo sobre las motivaciones y dificultades para estudiar la carrera; se concluye que esta es una reflexión pendiente por problemas en la comunicación de la profesión, el conocimiento de los aspirantes y los conflictos para armonizar cantidad y calidad de estudiantes.

Palabras Clave: Elección Profesional, Profesión, Bibliotecología, Archivística.

\section{Abstract}

This document checks over the reasons why library and archivist science are studied and the institutional strategies to attract students. It is started from a general research on the field that not only includes students, but also graduates, experts and employers; through a joint study that allows to know the opinion of this group about the motivations and difficulties of this career, where the conclusion is that this is a pending consideration caused by issues in communication about the field, knowledge of aspiring candidates and conflicts in reconciling quantity and quality of the student body.

Keywords: Professional Choice, Profession, Librarianship, Professional Archival. 


\section{Introducción}

Uno de los mayores desafíos que afrontan hoy los programas de Bibliotecología y Archivística son las escasas solicitudes de ingreso que reciben y que en incontables casos han puesto en peligro su subsistencia; el presente texto aborda un tema central de las instituciones educativas, captar nuevos estudiantes, a partir del conocimiento de los motivos de elección profesional.

El desarrollo se realiza a partir de la investigación sobre la bibliotecología como profesión en Colombia que analizó los elementos que la caracterizan como profesión y sus ciclos de desarrollo; además, la identidad profesional para describir cómo se ha dado su proceso de socialización, y las diferentes dinámicas y relaciones que ha establecido a través de su ejercicio profesional, para con todo ello comprender la realidad técnica y social que la dimensionan como profesión; la elección profesional se aborda teniendo en cuenta la perspectiva de Dubar (2000) que asevera que el momento de la elección de carrera es un aspecto clave en la construcción de "la identidad profesional de base", ya que obliga al sujeto a replantearse y reafirmar las formas identitarias subjetivas y objetivas construidas hasta ese momento y a imaginar una proyección laboral, económica, relacional y personal a partir de la dedicación de tiempo e interés en el estudio de un área.

En esa medida, la elección de una carrera no implica tan solo la búsqueda de un diploma o de un grado académico, sino también $y$, principalmente, la construcción personal de una estrategia identitaria a partir del autoconcepto y del reconocimiento social de los sujetos significativos y el conocimiento.

\section{Contexto teórico}

En la revisión teórica fue posible determinar que en la elección profesional las influencias existentes son diversas para cada individuo, responden a distintos factores y no tienen que ver con un solo motivo. Se presentan, entre otras: personales y/o de orientación vocacional, 
recomendación de amigos o conocidos, seguridad y crecimiento en el trabajo, suerte o casualidad, tradición familiar, oportunidades en el mercado laboral, buena remuneración y mejora en los ingresos económicos, mayores salidas para las personas del mismo género, orientación sexual o las dos a la vez, posibilidad ante la no admisión para los estudios de preferencia o el gusto específico por algún aspecto de la carrera, costos de la profesión y facilidad con que se percibe.

Es de anotar que, para la mayoría de los autores, los intereses y preferencias vocacionales son el elemento más diferenciador y decisivo en la elección. Al respecto, Rivas y Pascual (2003), elaboraron el Inventario de Factores Vocacionales (IFV) para delimitar el influjo de los elementos que afectan a la conducta vocacional; a partir de este inventario puede observarse que existen aspectos sociales, enfoques psicológicos y características de oportunidad que motivan la elección. Entre las razones de carácter social están: los condicionantes familiares (Influencia del padre, de la madre y / o de algún familiar, ambiente de casa, situación económica familiar, presión familiar respecto a la selectividad) aunque no hay que dejar de lado aspectos socioeconómicos como la etnia, sexo, edad y familia: profesión de los padres, número de hermanos, actitudes familiares, recursos.

También, los estereotipos sociales (poder, prestigio, salidas profesionales, prestigio social de los estudios, poder económico y social que se quiere conseguir). $Y$ los condicionantes académicos (dificultad, facilidad, duración de los estudios, horario y tiempo disponible, estudiar en la proximidad de casa) aunque hay que considerar además dimensiones pedagógicas como la percepción de estudios fáciles o la existencia de espacios académicos que resultan de interés, sin dejar de lado la oferta de carreras profesionales, las condiciones de ingreso y los costos de las matrículas.

Ahora bien, desde el enfoque psicológico pueden ser: la madurez vocacional (expectativas de éxito en los estudios elegidos, percepción de control sobre los acontecimientos, gustos y preferencias, éxito en materias relacionadas con la opción vocacional elegida, autoconocimiento, conocimiento del mundo laboral al que se dirige, constancia, capacidad de 
trabajo y sacrificio. Otro factor psicológico relevante es el asesoramiento vocacional (asesoramiento vocacional recibido, haber conocido a una persona relevante del área vocacional elegida, ser una carrera que se ajuste a la condición de hombre o mujer, apoyo, ayuda, estímulo de algún profesor).

En cuanto a los factores de oportunidad, tenemos la preparación para la oportunidad (alerta para aprovechar, en función del tiempo disponible y economía familiar, las posibilidades que se cruzan en el camino personal, la preparación personal a lo largo de los años, aprovechando las oportunidades de formación que se han presentado, la suerte). Otro elemento es la oportunidad de formación/ proyección (alerta y aprovechamiento de las posibilidades de formación novedosas, en lo que respecta a sus salidas profesionales y a la confianza personal en desarrollar con éxito tales estudios, así como las profesiones que implican, la casualidad).

Para Rubio (2006), la elección de carrera representa una extensión de la personalidad y una tentativa de implantar el estilo de comportamiento personal en el contexto de la vida laboral. Las personas proyectan sobre los títulos ocupacionales sus puntos de vista acerca de ellas mismas y del mundo laboral que prefieren. En este sentido, las expectativas laborales y la satisfacción del individuo con la profesión hacia la que se encamina se encuentran entre los factores más importantes a la hora de determinar las preferencias profesionales, por lo cual Martínez-Martínez (2013) sugiere analizar exhaustivamente las destrezas y habilidades del sujeto. Otros aspectos por considerar los refiere Cardona (2012): las posibilidades o dificultades económicas del núcleo familiar, la confusión sobre el futuro, la presión socio familiar y las ofertas educativas del lugar en el que se vive.

Castañeda y Solorio (2014) señalan que diversas investigaciones han hecho hincapié en las implicaciones de la elección de una institución en relación con las identidades individuales y profesionales, las posibilidades de adaptación institucional, las expectativas laborales, afirmando que sin importar los estudios de pertinencia que se realizan para la apertura de nuevas carreras, un importante porcentaje de los alumnos y alumnas en los centros 
universitarios regionales realizan la elección profesional, no de acuerdo con su vocación, sino con la oferta institucional presente en su región.

En el caso de la Bibliotecología y Archivística, existen variadas investigaciones que intentan explicar desde diversas aristas los factores que inciden en la elección profesional; en el caso de la vocación, según Fernández (1991, p.43), "la vocación por la bibliotecología es una vocación tardía, se da cuando hemos trabajado en una biblioteca o bien cuando al ingresar a la universidad, no queda más que esta opción porque las demás carreras tradicionales están saturadas". Esto es confirmado en el estudio realizado por De la Mano y Moro (2014), que encontraron que hay un marcado interés por el ejercicio de la profesión y no hay un fuerte factor vocacional; así mismo se destaca la influencia de la percepción social de la profesión.

Para Escalona y Tejada (2016) el perfil de ingreso va más allá de las motivaciones que tiene el alumno al elegir una carrera; el perfil es el conjunto de conocimientos, habilidades, aptitudes y actitudes que debe tener el aspirante, lo cual es diferente de los requisitos administrativos necesarios para ingresar a una carrera profesional. Al respecto Gardner (2005) plantea la importancia de que cada persona conozca sus habilidades, destrezas y capacidades, lo que permitiría conocer el tipo de inteligencia que predomina (dentro de la teoría de inteligencias múltiples), ubicando la bibliotecología dentro de la inteligencia visual/espacial. Ander-Egg (2006) señala que las características que se desarrollan en esta inteligencia son: habilidad para crear un modelo mental del mundo en tres dimensiones, capacidad de transformar imágenes, comprensión de ver el espacio como un todo, capacidad de reconocer el mismo objeto en diferentes perspectivas, anticiparse a las consecuencias de los cambios espaciales.

González y Álvarez (2009) en su investigación sobre la orientación vocacional, encontraron que no se tiene claro el campo de acción o de trabajo y que el conocimiento inicial acerca de la carrera fue a través de familiares y amistades. Algo similar ocurre con el estudio de Vallejo (2010), en cuanto a la necesidad de tener algún aprendizaje o conocimientos para ejercer en la bibliotecología, en su estudio pudo observar la idea de los estudiantes de secundaria de no 
requerir formación o incluso necesitar aprendizajes básicos, solo asociadas a estudiar o trabajar con el libro, sin haber ningún acercamiento con otra diversidad de formatos y mucho menos con las tecnologías de la información y la comunicación. Esto claramente está asociado a los deficientes servicios de orientación profesional en Bibliotecología y Archivística, en los que en muchas ocasiones no se incluyen en guías ni en programas desarrollados en los colegios, con lo cual como lo esboza Newbutt, (2012) es un factor importante para que la bibliotecología no llame la atención de los jóvenes.

El estudio de Hussey (2009) exploró las motivaciones de los estudiantes americanos afros, hispanos, asiáticos y nativos en la elección de la bibliotecología como carrera. Sus principales conclusiones se enfocan a que el uso de la biblioteca desempeña el papel más importante en la elección de la bibliotecología como profesión. El entorno de la biblioteca se percibe como un espacio seguro, cómodo e inclusivo. A esto le siguen las experiencias de trabajo de la biblioteca, el que los bibliotecarios fueron raramente mencionados como una influencia y que se reconociera la biblioteca como institución cultural blanca. Sin embargo, en el estudio realizado por Remy (2011) sobre la exploración de la cultura social de la bibliotecología, se encontró que las razones de la elección de la bibliotecología como carrera parecían no tener relación con la identidad y las potencialidades sociales de trabajar en la biblioteca. Con lo cual el autor sugiere que puede haber potencial para reclutar más estudiantes si el enfoque social de la profesión se proyecta de manera más eficaz.

\section{Objetivos}

La investigación de Vallejo (2014), de la que parte este documento tuvo como objetivo general, comprender el proceso de profesionalización de la bibliotecología en Colombia a través de los grupos sociales que establecen el campo profesional y uno de sus objetivos específicos se concentró en describir el proceso de socialización de la bibliotecología, en el cual se interiorizan los valores y conocimientos de la profesión, específicamente la identidad profesional donde uno de los criterios estudiados fue la elección de la carrera. 


\section{Metodología}

Por las características de este estudio, se consideró conveniente un diseño metodológico mixto, cualitativo y cuantitativo. La población por investigar fue: expertos (presidentes de agremiaciones profesionales y miembros de la academia, así como directores de escuelas), empleadores (por tipo de bibliotecas), profesionales egresados y estudiantes de las diferentes escuelas. Para cada uno de estos grupos, se diseñaron muestras e instrumentos de recolección de información de acuerdo con sus características propias. Por consiguiente, en los estudiantes (235) y profesionales (322) se establecieron muestras guía; en los empleadores (8), una muestra intencionada, y en las agremiaciones y escuelas, una muestra de expertos (22). Con ellos se establecieron técnicas e instrumentos de recolección de la información como el método Delphi, la entrevista y la encuesta. Las entrevistas se realizaron en las ciudades de Bogotá y Medellín, y las encuestas en línea se aplicaron en todo el país.

El procesamiento de los datos se hizo primero a partir de los resultados por grupo y posteriormente por cada una de las categorías de análisis y, finalmente, hacer un análisis en conjunto, de manera que se diera una comprensión más amplia y profunda del proceso de profesionalización de la bibliotecología en Colombia. El análisis de los datos cualitativos se realizó de acuerdo con el proceso definido por Martínez (2007) y el esquema planteado por Strauss y Corbin (2002). El procesamiento de los datos cuantitativos se realiza de acuerdo con las medidas estadísticas.

\section{Resultados}

De acuerdo con los expertos en su parte cualitativa, las motivaciones para elegir la bibliotecología como profesión son de carácter social y los condicionantes académicos como se observa en la Cuadro 1. 


\section{Cuadro 1 \\ Motivaciones para elegir la bibliotecología como profesión/ expertos}

\begin{tabular}{|c|c|}
\hline $\begin{array}{l}\text { "Profesionalizarse para poder ascender dentro de } \\
\text { la pirámide laboral". (E24,P20) }\end{array}$ & $\begin{array}{l}\text { "La percepción de ser una profesión fácil } \\
\text { de cursar". (E24,P20) }\end{array}$ \\
\hline $\begin{array}{l}\text { "Porque efectuaron algún tipo de práctica } 0 \\
\text { pasantía en una biblioteca y les quedó gustando. } \\
\text { Porque los primeros trabajos que consiguieron fue } \\
\text { en alguna Biblioteca, les ha gustado y ahora } \\
\text { buscan su profesionalización". (E7,P20) }\end{array}$ & $\begin{array}{l}\text { "La elección muchas veces se hace } \\
\text { porque las exigencias en cuanto al } \\
\text { puntaje del examen de admisión son } \\
\text { menores que los exigidos para otras } \\
\text { carreras". (E6,P20) }\end{array}$ \\
\hline $\begin{array}{l}\text { "Una de las principales motivaciones es la } \\
\text { consecución rápida de un trabajo. A esto se } \\
\text { agrega, los relativamente bajos costos de la } \\
\text { matrícula". (E12,P20) }\end{array}$ & $\begin{array}{l}\text { "La pasión por la lectura, el interés por el } \\
\text { conocimiento, mantenerse actualizado y } \\
\text { apoyar con información en sus procesos } \\
\text { de investigación". (E8,P20) }\end{array}$ \\
\hline $\begin{array}{l}\text { "Actualmente las personas que escogen } \\
\text { bibliotecología como profesión es porque se } \\
\text { encuentran trabajando en el área o tienen } \\
\text { familiares profesionales bibliotecólogos, algunos } \\
\text { se inclinan por la buena oportunidad laboral en } \\
\text { términos económicos que represente esta } \\
\text { carrera". (E22,P20) }\end{array}$ & $\begin{array}{l}\text { "Convicción profunda del valor que esta } \\
\text { profesión tiene para la sociedad y una } \\
\text { visión prospectiva del papel que puede } \\
\text { jugar en el desarrollo de nuestro país". } \\
(\mathrm{E} 1, \mathrm{P} 20)\end{array}$ \\
\hline $\begin{array}{l}\text { "La poca existencia de profesionales graduados } \\
\text { en el área". }(E 15, P 20)\end{array}$ & $\begin{array}{l}\text { "Porque es la carrera de otros familiares } \\
0 \quad \text { personas muy cercanas } \\
\text { afectivamente". (E7,P20) }\end{array}$ \\
\hline
\end{tabular}

Fuente: elaboración propia

Las motivaciones para elegir la bibliotecología como profesión son en su mayoría de carácter económico, pedagógicas y familiares. En cuanto a las de carácter económico y laboral, pueden resaltarse la búsqueda de seguridad y crecimiento en el trabajo, la existencia de pocos profesionales en el área, aunque preferiblemente es por su mercado laboral, salario, ingresos económicos o costos de la profesión. En cuanto a las motivaciones de índole pedagógica se resalta el que no es tan difícil como otras carreras, el gusto por la lectura, por el contenido e impacto de la profesión. Ahora bien, en su parte cuantitativa (ver Cuadro 2), se pueden observar las variaciones en las dos fases de búsqueda de consensos. En la fase 2 el nivel de consenso bajó de $50 \%$ a $31,8 \%$, así, la principal motivación para elegir la 
bibliotecología como profesión, según la opinión de los expertos, sigue siendo el mercado laboral, el salario, los ingresos económicos y los costos de la profesión; la segunda motivación más mencionada en ambas fases es el contenido e impacto de la profesión.

\section{Cuadro 2}

\section{La principal motivación para elegir la bibliotecología como profesión/expertos}

\begin{tabular}{|l|c|l|l|c|}
\hline \multicolumn{1}{|c|}{ Resultados Fase 1 } & $\begin{array}{c}\text { Porcentaje } \\
\text { de } \\
\text { selección }\end{array}$ & $9,1 \%$ & Resultados Fase 2 & $\begin{array}{c}\text { Porcentaje } \\
\text { de } \\
\text { selección }\end{array}$ \\
\hline El gusto por la lectura & $\begin{array}{l}\text { Por buscar seguridad y } \\
\text { crecimiento en el trabajo y } \\
\text { profesionalizarse }\end{array}$ & $4,5 \%$ \\
\hline $\begin{array}{l}\text { Porque no es tan difícil } \\
\text { como otras carreras }\end{array}$ & $13,6 \%$ & Por tradición familiar & $9,1 \%$ \\
\hline $\begin{array}{l}\text { Por la existencia de pocos } \\
\text { profesionales en el área }\end{array}$ & $13,6 \%$ & $\begin{array}{l}\text { Por la existencia de pocos } \\
\text { profesionales en el área }\end{array}$ & $9,1 \%$ \\
\hline Por tradición familiar & $18,2 \%$ & El gusto por la lectura & $9,1 \%$ \\
\hline $\begin{array}{l}\text { Por buscar seguridad y } \\
\text { crecimiento en el trabajo y } \\
\text { profesionalizarse }\end{array}$ & $22,7 \%$ & $\begin{array}{l}\text { Porque no es tan difícil } \\
\text { como otras carreras }\end{array}$ & $13,6 \%$ \\
\hline $\begin{array}{l}\text { Por el contenido e impacto } \\
\text { de la profesión }\end{array}$ & $22,7 \%$ & $\begin{array}{l}\text { Por el contenido e } \\
\text { impacto de la profesión }\end{array}$ & $22,7 \%$ \\
\hline $\begin{array}{l}\text { Por su mercado laboral, } \\
\text { salario, ingresos } \\
\text { económicos o costos de la } \\
\text { profesión }\end{array}$ & $50,0 \%$ & $\begin{array}{l}\text { Por su mercado laboral, } \\
\text { salario, ingresos } \\
\text { económicos o costos de } \\
\text { la profesión }\end{array}$ & $31,8 \%$ \\
\hline Fuente: & & \multicolumn{2}{|l}{} \\
\hline
\end{tabular}

Fuente: elaboración propia.

En el caso de los empleadores, los principales motivos son la vocación, la recomendación de amigos y familiares, las experiencias con bibliotecas, las oportunidades laborales e ingresos, que no tiene que ver con las matemáticas y que su elección no se da por moda, como se observa en el Cuadro 3. 


\section{Cuadro 3 \\ Motivos de elección profesional/empleadores}

\begin{tabular}{|c|c|}
\hline Vocación & $\begin{array}{l}\text { "por no decir todos los chicos que hoy día trabajan en nuestra } \\
\text { biblioteca pasaron por alfabetizadores acá, o sea que si hay un } \\
\text { proceso de formación adecuado y de cultura adecuada frente a la } \\
\text { biblioteca y desde el conocimiento, desde la etapa preescolar, primaria } \\
\text { y una buena impresión se llega a poder tener la opción de escoger esta } \\
\text { profesión desde la vocación, pero pues como no llegamos a muchas } \\
\text { partes". (EM6,L227) }\end{array}$ \\
\hline $\begin{array}{l}\text { Recon } \\
\text { amigo }\end{array}$ & $\begin{array}{l}\text { "logrado que algunos familiares o inclusive amistades estudien } \\
\text { bibliotecología". (EM1,L70) }\end{array}$ \\
\hline $\begin{array}{r}\text { Experie } \\
\text { biblic }\end{array}$ & $\begin{array}{l}\text { Claro, que aprendieron a hacer unas cositas y luego se meten a la } \\
\text { universidad para acabarlas de organizar, vienen con eso que } \\
\text { aprendieron a hacer allá prestando el servicio social, de alfabetizador, } \\
\text { o prestando el servicio en la biblioteca popular, o haciendo una práctica } \\
\text { y de pronto les sonó y se entraron". (EM4,L490) } \\
\text { ".... porque hay una imagen de que solo el que fue bibliotecario por } \\
\text { oficio puede llegar a ser bibliotecólogo de profesión, entonces sí tiene } \\
\text { que ver mucho con la experimentación, y con el haber tenido un } \\
\text { contacto bueno". (EM6, L251) }\end{array}$ \\
\hline $\begin{array}{r}\text { Opor } \\
\text { laborale }\end{array}$ & $\begin{array}{l}\text { "el mercado laboral está pulpito, yo a todos los jóvenes que puedo les } \\
\text { digo estudien bibliotecología, pero como en el imaginario de la gente } \\
\text { creen que el bibliotecólogo es alcanzar libros, entonces la gente dice } \\
\text { hay no que hartera estudiar eso para ir a cuidar libros". (EM3,L273) } \\
\text { "si dijéramos de un ciento por ciento las personas que ingresan por } \\
\text { vocación sería un } 30 \% \text {, el resto son personas que buscan asegurar } \\
\text { una carrera universitaria y una fuente de ingreso". (EM6,L95) }\end{array}$ \\
\hline No tie & "estudiar nada que tenga que ver con matemática". (EM1,L303) \\
\hline $\begin{array}{l}\text { No se elige por } \\
\text { moda }\end{array}$ & $\begin{array}{l}\text { "... la bibliotecología no es una profesión chévere, ni es chévere, ni es } \\
\text { play, ni es nada de esas cosas, ya, tú te imaginas una cosa muy } \\
\text { académica, muy de leer, la gente no lee, los libros no son un objeto } \\
\text { importante, en cuántas casas por qué no hacen censo de en cuántas } \\
\text { casas hay libros, pregúntele a los bibliotecólogos si en las casas de } \\
\text { ellos hay libros por ejemplo". (EM2,L269) }\end{array}$ \\
\hline
\end{tabular}

Fuente: elaboración propia. 
Por otra parte, los empleadores destacan otros elementos por tener en cuenta como los deficientes procesos de selección, porque no hay claridad de los perfiles de ingreso y la valoración y el reconocimiento social de la profesión:

"Hacer procesos de selección, si siguen dejando entrar a todo el mundo a estudiar bibliotecología es bien difícil. Yo pienso que lo primero que toca perfilar es, cuál es el estilo de estudiante y de profesional que nosotros queremos sacar de la Universidad... porque no puede ser que todo el mundo tenga las competencias para ser bibliotecólogo, y no puede ser que por llenar las facultades de bibliotecología entre todo el mundo para cumplir con unas metas". (EM1,L244)

"Si tenemos buenos profesores y tenemos un proceso de filtro y decantación al ingreso de la carrera vamos a tener buenos profesionales y gente convencida, no gente necesitada". (EM1,L257)

“¿Yo estudio bibliotecología porque quiero ser el dirigente de este país?, o ¿estudio bibliotecología porque yo quiero ser un experto en procesamientos, sistemas de información, alfabetización informática o informacional?, bueno lo que sé”. (EM7, L560)

En cuanto a los profesionales, frente a la pregunta sobre qué piensan al ser hoy bibliotecólogos, los resultados fueron los siguientes: a usted le gusta sentirse un bibliotecólogo ahora $(86,5 \%)$; obtener el título profesional contribuyó a cumplir sus expectativas y sueños $(79,63 \%)$; si volviera a empezar y con la experiencia que tiene elegiría de nuevo ser bibliotecólogo (74,14\%); y en las actuales condiciones profesionales, le recomendaría a su hijo/hija o un familiar que estudiara bibliotecología, mínimo el $60 \%$ de los profesionales responden con seguridad que realmente es algo que experimentan como bibliotecólogos. Para las demás afirmaciones la opinión se divide entre las definidas "con dudas" o "de ninguna manera, ver Cuadro 4.

Resulta interesante que para el $58,81 \%$ a través de la bibliotecología haya logrado movilidad social, aunque los que dudan y los que creen que no, suman el $41,19 \%$. Por otra parte, para los que tenían la bibliotecología como su primera opción a la hora de realizar los estudios universitarios, sumando los que tienen dudas o no la tenían como primera opción, obtenemos 
el $64,99 \%$, aunque el $74,14 \%$ volviera a empezar y con la experiencia que tienen elegirían de nuevo ser bibliotecólogos. El 36,61\% dudan o no recomendarían a su hijo/hija o un familiar que estudiara bibliotecología y el $50,34 \%$ no tenían sus intereses profesionales claros al elegir la profesión de bibliotecólogo.

Cuadro 4

Ser bibliotecólogo hoy/profesionales

\begin{tabular}{|l|c|c|c|c|c|c|c|c|}
\hline \multicolumn{1}{|c|}{ Afirmaciones } & \multicolumn{2}{|c|}{$\begin{array}{c}\text { Con } \\
\text { seguridad }\end{array}$} & \multicolumn{2}{c|}{ Con dudas } & \multicolumn{2}{c|}{$\begin{array}{c}\text { De ninguna } \\
\text { manera }\end{array}$} & \multicolumn{2}{c|}{ No } \\
& responde \\
\cline { 2 - 11 } & No. & $\%$ & No. & $\%$ & No. & $\%$ & No. & $\%$ \\
\hline $\begin{array}{l}\text { A usted le gusta sentirse un bibliotecólogo } \\
\text { ahora }\end{array}$ & 378 & 86,5 & 51 & 11,67 & 8 & 1,83 & 0 & 0 \\
\hline $\begin{array}{l}\text { Obtener el título profesional contribuyó a } \\
\text { cumplir sus expectativas y sueños }\end{array}$ & 348 & 79,63 & 71 & 16,25 & 18 & 4,12 & 0 & 0 \\
\hline $\begin{array}{l}\text { De acuerdo con las condiciones del } \\
\text { ejercicio profesional de la bibliotecología, } \\
\text { esta ha sido un vehículo de movilidad } \\
\text { social (escalar en el estrato social) }\end{array}$ & 257 & 58,81 & 108 & 24,71 & 72 & 16,48 & 0 & 0 \\
\hline $\begin{array}{l}\text { Fue la bibliotecología su primera opción a } \\
\text { la hora realizar los estudios universitarios }\end{array}$ & 153 & 35,01 & 81 & 18,54 & 203 & 46,45 & 0 & 0 \\
\hline $\begin{array}{l}\text { Si volviera a empezar y con la experiencia } \\
\text { que tiene elegiría de nuevo ser } \\
\text { bibliotecólogo }\end{array}$ & 324 & 74,14 & 79 & 18,08 & 33 & 7,55 & 1 & 0,23 \\
\hline $\begin{array}{l}\text { En las actuales condiciones profesionales, } \\
\text { le recomendaría a su hijo/hija o un familiar } \\
\text { que estudiara bibliotecología }\end{array}$ & 277 & 63,39 & 111 & 25,4 & 49 & 11,21 & 0 & 0 \\
\hline $\begin{array}{l}\text { Sus intereses profesionales estaban claros } \\
\text { al elegir la profesión de bibliotecólogo }\end{array}$ & 217 & 49,66 & 145 & 33,18 & 75 & 17,16 & 0 & 0 \\
\hline
\end{tabular}

Fuente: elaboración propia

Ahora bien, al evaluar, ¿cómo le ha resultado el haber elegido la carrera de Bibliotecología?, para esta pregunta es claro que el resultado ha sido positivo en todos los sentidos, para el $69,34 \%$ de los profesionales. El resto de las opciones no fueron elegidas por más del $12 \%$ de los profesionales. 
Para los estudiantes, las principales motivaciones que los llevaron a elegir estudiar bibliotecología son los motivos personales u orientación vocacional (53,88\%). Para el resto de las opciones planteadas no son fuertes motivaciones para la elección: la recomendación de amigos o conocidos, la suerte o casualidad y la tradición familiar, $69,83 \%$ de los estudiantes. Tampoco fueron relevantes en la selección que la profesión no fuera tan difícil como otras carreras, o porque no los aceptaban en los estudios que preferían, o porque tiene más salidas para las personas del mismo género u orientación sexual, ver Cuadro 5.

\section{Cuadro 5}

\section{Motivaciones para elegir Bibliotecología/estudiantes}

\begin{tabular}{|l|c|c|}
\hline \multirow{2}{*}{ Motivaciones } & \multicolumn{2}{c|}{$\begin{array}{c}\text { Porcentaje de } \\
\text { estudiantes }\end{array}$} \\
\cline { 2 - 3 } & Sí & No \\
\hline Su elección fue por motivos personales u orientación vocacional & 53,88 & 46,12 \\
\hline Su elección fue por recomendación de amigos o conocidos & 30,17 & 69,83 \\
\hline Elegí la bibliotecología para buscar seguridad y crecimiento en el trabajo & 26,72 & 73,28 \\
\hline Elegí la bibliotecología por suerte o casualidad & 10,34 & 89,66 \\
\hline Elegí bibliotecología por tradición familiar & 3,88 & 96,12 \\
\hline $\begin{array}{l}\text { Elegí esta carrera por su mercado laboral, salario, ingresos económicos o } \\
\text { costos de la profesión }\end{array}$ & 32,33 & 67,67 \\
\hline Elegí bibliotecología porque no es tan difícil como otras carreras & 1,72 & 98,28 \\
\hline Elegí bibliotecología porque no me aceptaban en los estudios que prefería & 4,74 & 95,26 \\
\hline $\begin{array}{l}\text { Elegí esta carrera porque tiene más salidas para las personas de mi } \\
\text { mismo género u orientación sexual }\end{array}$ & 2,16 & 97,84 \\
\hline Elegí esta carrera por mi gusto hacia la lectura & 37,07 & 62,93 \\
\hline Otro & 7,76 & 92,24 \\
\hline \multicolumn{2}{|c|}{ TOTAL } & $\mathbf{1 0 0 \%}$ \\
\hline
\end{tabular}

Fuente: elaboración propia

De otra parte, llama la atención que el $62,93 \%$ de los encuestados no eligieran la carrera por el gusto por la lectura. Si tomamos en cuenta dos aspectos relacionados con el entorno laboral (la búsqueda de seguridad y el crecimiento en el trabajo $(26,72 \%$ ), y el mercado laboral, salario, ingresos económicos o costos de la profesión $(32,33 \%)$, se observa que sumándolos se llegaría a un $59,05 \%$ de representatividad. 
El 54,31\% de los estudiantes consideran que la elección de la carrera de Bibliotecología ha resultado positiva en todos los sentidos. Los resultados frente a las dificultades que ha afrontado al haber elegido la carrera de Bibliotecología muestran que la principal es que otros profesionales están a cargo de las bibliotecas $(32,76 \%)$ y el desconocimiento de la titulación y competencias por la sociedad (ver Cuadro 6).

\section{Cuadro 6}

\section{Principal dificultad que ha afrontado al haber elegido la carrera de} Bibliotecología/estudiantes

\begin{tabular}{|l|c|c|}
\hline \multirow{2}{*}{ DIFICULTADES } & \multicolumn{2}{c|}{$\begin{array}{c}\text { Porcentaje de } \\
\text { estudiantes }\end{array}$} \\
\cline { 2 - 3 } & Sí & No \\
\hline Baja valoración a los temas de la información y las bibliotecas & 25,43 & 74,57 \\
\hline Bajos salarios asignados & 24,14 & 75,86 \\
\hline Deficiencias en la formación & 18,1 & 81,9 \\
\hline Gremios débiles & 15,52 & 84,48 \\
\hline Estereotipo social del bibliotecólogo & 20,69 & 79,31 \\
\hline Desconocimiento de la titulación y competencias por la sociedad & 31,03 & 68,97 \\
\hline Bajo prestigio y reconocimiento profesional & 20,26 & 79,74 \\
\hline Oportunidades laborales exclusiva al sector de bibliotecas & 10,78 & 89,22 \\
\hline Justificación de su elección ante su círculo social y familiar & 3,88 & 96,12 \\
\hline Otros profesionales están a cargo de las bibliotecas & 32,76 & 67,24 \\
\hline Ninguna & 9,91 & 90,09 \\
\hline Otro & 3,88 & 96,12 \\
\hline \multicolumn{2}{|c|}{$\mathbf{1 0 0} \%$} \\
\hline
\end{tabular}

Fuente: elaboración propia

Resulta interesante que los estudiantes no hayan tenido dificultades frente a inconvenientes como: la baja valoración de los temas de la información y las bibliotecas, los bajos salarios asignados, las deficiencias en la formación, los gremios débiles, el estereotipo social del bibliotecólogo, el bajo prestigio y reconocimiento profesional, las oportunidades laborales exclusivas en el sector de bibliotecas, la justificación de su elección ante su círculo social y familiar; porque puede suponer un avance significativo en los estereotipos que siempre han acompañado la profesión. 


\section{Estrategias institucionales}

Los programas de Bibliotecología y Archivística en muchos lugares del mundo han empezado a tener dificultades para captar estudiantes, las razones pueden estar asociados a diferentes aspectos: variada oferta de carreras, dificultades económicas, ausencia de programas de orientación profesional, canales de comunicación inadecuados para el público interesado, poco interés por la profesión asociada aún a estereotipos, políticas educativas que privilegian algunas áreas de conocimiento; pero tal vez la dificultad mayor tiene que ver con el desarrollo de estrategias específicas para los programas del área, conociendo los perfiles de quienes estudian, brindando información oportuna para los interesados.

A primera vista, estos estudios se realizan para conocer las motivaciones que tuvieron los aspirantes para elegir la Bibliotecología y la Archivística como profesión; sin embargo, son una base importante para repensar la manera en cómo se está invitando a estudiarla; ocurre que el papel de promoción es asumido en la mayor parte de los casos por oficinas centrales de las universidades que generalmente no cuentan con los conocimientos adecuados para hablar de la profesión y su proyección actual. Los resultados nos demuestran que el proceso de selección para esta carrera difiere de las razones convencionales, por lo cual es indispensable diseñar una estrategia diferente y única que debería tener además de las usadas en la universidad para las demás carreras, elementos como:

- Mensaje y canal de comunicación

- Conocimiento del perfil de los estudiantes

- Armonización entre cantidad y calidad

\section{a. Comunicación}

El tema de la comunicación de la profesión debe ser abordado desde dos caminos, el mensaje que estamos transmitiendo y los canales que estamos utilizando para ello. Hoy es importante preguntarnos, cuáles son los valores agregados que tiene estudiar Bibliotecología y Archivística, toda vez que como lo arrojan los resultados, los criterios por los cuales se elige 
ser bibliotecólogo y archivista están desligados de la naturaleza interna de la profesión. Otro factor por tener en cuenta es que la mayor parte de los profesionales que hicieron parte del proyecto no tenían claros sus intereses profesionales y los estudiantes no asumían dentro de sus objetivos al formarse para ser bibliotecólogos el "comprender la cultura profesional y los valores".

Un ejemplo del mensaje se da al revisar los enfoques que le dan a la profesión, se hizo una búsqueda en Google sobre, por qué estudiar Bibliotecología, y se encontró la siguiente información:

- 'La Bibliotecología es la profesión que forma a las personas que se ocuparán de administrar las bibliotecas públicas, privadas, escolares y universitarias con los conocimientos necesarios para proporcionar la información que cada asociado requiera"1.

- "La Bibliotecología se fundamentan en la formación teórica y metodológica necesaria para la organización, sistematización y clasificación de la información bibliográfica, la formación de colecciones documentales en medios tanto impresos como electrónicos; la organización de sistemas bibliotecarios y la gestión de servicios para el uso de información de libros, revistas y otros medios de publicación"2.

- "Se enfoca en la organización, sistematización y clasificación de la información bibliográfica, así como en el establecimiento de colecciones documentales tanto en medios impresos como electrónicos. Se encarga de la organización de sistemas y gestión de servicios para el uso de información de libros, revistas y otros medios de publicación para que los usuarios accedan fácil, rápidamente, eficazmente a la información. Esta carrera se encarga de la restauración y el cuidado de archivos, documentos, escritos, y demás objetos con valor histórico y cultural”3.

\footnotetext{
${ }^{1}$ https://www.nosequeestudiar.net/carreras/bibliotecologia/por-que-estudiar-bibliotecologia/

${ }^{2}$ https://orientacion.universia.net.co/carreras_universitarias/bibliotecologia-30.html

${ }^{3}$ http://www.guiaacademica.com/educacion/pregrado/carreras/bibliotecologia.aspx
} 
¿Estos mensajes representan lo que es hoy la profesión?, ¿realmente es motivante para alguien acercarse a la profesión a partir de estas descripciones?; evidentemente el lenguaje utilizado está dirigido a personas que conozcan el mundo de las bibliotecas y los archivos; además se está partiendo del supuesto que está enfocada al mundo de las bibliotecas y los archivos históricos, aunque los perfiles laborales de la mayor parte de las escuelas están mostrando otras salidas laborales.

Por otra parte, en la información que presentan las páginas web de algunas escuelas, es clara su orientación a lo institucional, por lo cual carecen de un lenguaje fresco, personal e informal dirigido a los aspirantes. No es posible encontrar contenidos que el aspirante pueda leer para conocer la profesión, por ejemplo. Incluso otros elementos que pueden jugar en la selección de la profesión: la acreditación de alta calidad y lo que esto significa para un estudiante, el tiempo que va a tomar en graduarse; el tiempo que le toma en promedio ubicarse laboralmente, las posibilidades de prácticas profesionales y los lugares más destacados donde se realizan, las opciones de doble titulación, las opciones de programas de intercambio, lo que esto implica y los lugares, el prestigio y reconocimiento de la institución y sus profesores.

Todo esto nos lleva a reenfocar lo que queremos que se sepa de la profesión, vinculando la universidad y el programa con los intereses de los estudiantes, un caso exitoso de este tipo de estrategia de marketing es el de la Facultad de Artes y Letras de Harvard: "Haz lo que amas para llegar a ser lo que quieres".

Otro aspecto por considerar son los canales de comunicación a través de los cuales debemos llegar a los futuros estudiantes, hoy siguen siendo comunes los folletos, las ferias educativas, las jornadas de puertas abiertas donde las posibilidades de llegar a los estudiantes son muy pocas, porque la bibliotecología y la archivística como pudimos observar en los resultados no son la primera opción al elegir una carrera; también se puede apreciar unas pobres prácticas de posicionamiento en la web así como también las redes sociales comúnmente usadas; si como lo arrojan los resultados, es posible identificar que hay una madurez personal e 
intelectual en la elección, pues esta fue especialmente por motivos personales o de orientación vocacional y por el entorno laboral de la profesión, es posible pensar en otros medios para comunicar la profesión.

Hay que revisar quién es el responsable dentro de los programas de captar estudiantes, o esto se deja al libre albedrío o la suerte; claramente toda la comunidad académica debe ser quien oriente y motive a estudiar la carrera, los directivos, profesores y egresados; especialmente aquellos reconocidos en el sector que pueden ser "influenciadores", otro aspecto relevante son los lugares más representativos donde ellos están ubicados, que pueden transmitir un mensaje de interés para los aspirantes.

\section{b. Conocimiento del perfil}

La pregunta que deberíamos formularnos es si conocemos ¿cómo llegaron los estudiantes a la universidad?, ¿cuáles fueron los criterios para elegir el programa y la institución educativa? Esta es una invitación a conocer los principales rasgos de los estudiantes que eligen la profesión, que es finalmente como se da un uso apropiado de este tipo de estudios; por ejemplo, los resultados nos arrojan que sigue siendo mayor el porcentaje de mujeres que ingresan, que el promedio de edad es de unos 25 años y la mayor parte de las personas que optan por la profesión tienen una experiencia de trabajo previa en el área. Esta información deberá ser profundizada para conocer los principales intereses, cómo les gusta relacionarse con la universidad y el programa, y cuáles son los mecanismos más comunes de comunicación y en general cuáles son las tendencias en la cultura estudiantil de los bibliotecólogos y archivistas.

Es importante tomar en cuenta que además de formarse como profesionales, los estudiantes aspiran a tener una experiencia integral en su paso por la universidad, pero además aspiran a mantener un vínculo permanente con el programa, para que haga parte de su vida profesional. Tal como lo expresa Gonzáles (2004) para la vocación médica, 
"es necesario que los objetivos educativos orientados al desarrollo de lo cultural y espiritual... El distanciamiento de estos principios... conduce a una enseñanza profesional en la que se tiende peligrosamente a enfatizar la información sobre la formación, la técnica sobre la compasión, la instrucción sobre la educación y la habilidad sobre la espiritualidad... Egresan así médicos cada vez más actualizados, pero menos sensibles; cada vez más técnicos, pero menos involucrados; cada vez más entrenados, pero menos disponibles; cada vez mejor equipados, pero menos integrales; cada vez más automatizados, pero menos humanizados; y cada vez más capaces de hacer, pero menos de ser". (párr. 21)

En este sentido, la decisión vocacional es un paso fundamental en la construcción de "la identidad profesional de base" esbozada por Dubar (2000), ya que se replantea y reafirma los imaginarios construidos hasta el momento y lleva a pensar en la proyección laboral, económica y personal, pues no solo se trata de obtener un título de bibliotecólogo y archivista, sino también de llegar a asumir lo que significa ser y actuar como tal.

\section{c. Armonizar cantidad y calidad}

La proyección de las escuelas de bibliotecología y archivística deben tener claro el tipo de estudiantes al que van a hacer su oferta académica, pensando en su realidad (los alumnos actuales) y el ideal (estudiantes que quisieran tener), esto sirve de orientación, y fortalecen la trayectoria en la universidad de los aspirantes. A partir de ello, es indispensable que las escuelas empiecen a combinar una estrategia que permita incrementar la cantidad de estudiantes, pero también mejorar la calidad de estos.

En virtud de ello, la universidad tiene una gran responsabilidad al definir los criterios de selección, para garantizar que las relaciones internas que se generen en el grupo ayuden a consolidar la identidad profesional, al ser integrado por personas con iguales intereses y apego por la profesión. Esto, por supuesto, de acuerdo con los mecanismos de ingreso de las escuelas de bibliotecología tiene dificultades para ser adoptado, pues en la mayoría de los 
casos no existe el mecanismo de entrevista, o existen criterios bajos para el ingreso, lo que hace que en algunos casos la elección no sea la indicada y lleva a que puedan ingresar personas que no tengan la vocación para ser bibliotecólogos o archivistas, desconozcan la profesión o incluso su interés sea exclusivamente laboral o por condiciones exclusivamente socioeconómicas. Las respuestas obtenidas en el estudio realizado confirman que la educación en bibliotecología colombiana es un mecanismo importante para lograr la movilidad social.

De todos modos, el aspirante que intenta ingresar a la universidad tiene claro qué es la profesión, lo cual muestra que en su etapa de estudiante no tendrá grandes dificultades con el descubrimiento de la bibliotecología y la archivística, tales que lo lleven a retirarse por ver defraudadas sus expectativas en relación con esta. Sin embargo, la dificultad mayor es formar personas en una profesión que no les atrae del todo.

\section{Referencias bibliográficas}

Ander-Egg, E. (2006). Claves para introducirse en el estudio de las inteligencias múltiples. Buenos Aires: Homo Sapiens.

Cardona, D. (2012). Prevalencia de intereses y preferencias profesionales en estudiantes de grado 11 de instituciones educativa públicas de la ciudad de lbagué. Revista Colombiana de Ciencias Sociales, 3 (2).

Castañeda, L.; Solorio, M. (2014). La elección de carrera: entre la vocación y las ofertas institucionales. Revista de Educación y Desarrollo, 28. Recuperado de http://www.cucs.udg.mx/revistas/edu desarrollo/anteriores/28/028 Castaneda.pdf

Dubar, C. (2000). La socialisation: construcciòn de identités sociales et professionelles. París: Armand Colin.

Escalona-Ríos, L. y Tejada-Artigas, C. (2016). Tan lejos, tan cerca: perfil y percepciones de los alumnos de primer curso de Bibliotecología de la UNAM y de la UCM hacia sus estudios. Investigación Bibliotecológica: Archivonomía, Bibliotecología e Información, 70. DOI: dx.doi.org/10.1016/j.ibbai.2016.10.008 
Fernández de Mora, R. (1991). El oficio del bibliotecólogo. Investigación Bibliotecológica, 5 (10).

Gardner, H. (2005). Inteligencias múltiples: la teoría en la práctica. Barcelona: Paidós.

González, M. y Álvarez, I. (2009). La orientación vocacional en los alumnos de ingreso a la Licenciatura en Bibliotecología y Estudios de la Información: una aproximación. Recuperado de http://ru.ffyl.unam.mx:8080/ispui/bitstream/10391/780/1/07 ABEI Gonzalez Alvarez 93 -105.pdf

Gonzáles, R. (2004). Lo cultural y lo espiritual en la formación médica: apreciaciones de estudiantes de 5to. año de Medicina. Rev Cubana Med Gen Integr, 20 (3), Recuperado de http://bvs.sld.cu/revistas/mgi/vol20 3 04/mgi02304.htm

Hussey, L. (2009). Why librarianship? An exploration of the motivations of ethnic minorities to choose library and information science as a career. En Delmus, W., James, N. y Janine, G. (ed.) Advances in Library Administration and Organization (Volume 28), Emerald Group Publishing Limited, pp. 153 - 217.

Martínez, M. (2007). La investigación cualitativa etnográfica en educación: manual teóricopráctico. México: Trillas.

Martínez-Martínez, A. (2013). La orientación como actividad educativa y vocacional en los itinerarios curriculares del alumnado de Bachiller y Formación Profesional y su inclusión en el mercado laboral. (Tesis Doctoral: Universidad de Granada). Recuperado de http://digibug.ugr.es/handle/10481/30837

De la Mano, M. y Moro, M. (2013). Motivaciones en la elección de la carrera universitaria: metas y objetivos de los estudiantes del Grado en Información y Documentación en la Universidad de Salamanca, en Investigación en Información. Documentación y Sociedad. Diálogos entre Brasil y España, A. Cuevas Cerveró y E. Simeão (coord.), 517-534. Madrid: Facultad de Ciencias de la Documentación. (Universidad Complutense). Recuperado de https://books.google.es/books?hl=es\&|r=\&id=wvj3AwAAQBAJ\&oi=fnd\&pg=PA185\&dq= elecci\%C3\%B3n+profesional\&ots=oQyc1t OVi\&sig=TU1m1YkYyrDhlFfg0Sw69 fzMWw $\# \mathrm{v}=$ onepage \&q=elecci\%C3\%B3n\%20profesional\&f=false 
Newbutt. S. (2012). ¿Qué impresiones tiene la gente sobre la bibliotecología como carrera profesional? Revista Interamericana de Bibliotecología, 35 (3). Recuperado de http://www.scielo.org.co/pdf/rib/v35n3/v35n3a7.pdf

Remy, B. (2011) Exploring the Social Culture of Librarianship through the Identifications and Experiences of Library Workers Who Are Members of Marginalized Social Groups. Recuperado de http://dagda.shef.ac.uk/dispub/dissertations/201011/External/BRemy zzz Formatted Dissertation done.pdf.

Rivas, F. y Pascual, F. (2003). Inventario de influjos/circunstancias vocacionales. En F. Rivas. (2003). Asesoramiento vocacional: teoría, práctica e instrumentación. Anexo Capítulo 1. Barcelona. Ariel

Rubio, N. (2006). Una propuesta de orientación vocacional para la educación media, diversificada y profesional venezolana. Buenos Aires: Fundación El Libro y Guía del Estudiante.

Strauss, A., y Corbin, J. (2002). Bases de la investigación cualitativa: técnicas y procedimientos para desarrollar la teoría fundamentada. Medellín: Universidad de Antioquia.

Vallejo-Sierra, R. (2010). Representaciones sociales de la bibliotecología: una mirada desde los jóvenes que culminaron los estudios secundarios. Revista Códice, 6 (1).

Vallejo-Sierra, R. (2014). La bibliotecología como profesión en Colombia. (Tesis Doctoral). Universidad de Salamanca.

\section{Notas de la autora}

Ruth Helena Vallejo Sierra: profesional en bibliotecología. Actualmente, se destaca como Investigadora independiente. Correo electrónico: rhvs2007@gmail.com

* Los datos que aquí se presentan y discuten se obtuvieron para la tesis "la bibliotecología como profesión en Colombia", presentada en la Universidad de Salamanca para obtener el título de Doctora en Metodologías y Líneas de Investigación en Biblioteconomía y Documentación. 